\title{
Vasculitis in children - a review
}

Volume 4 Issue 5 - 2016

\author{
AKM Mamunur Rashid \\ Department of Paediatrics, Khulna Medical College, Bangladesh
}

Abbreviations: KD, kawasaki disease; HSP, henochschonlein purpura; HIV, human immunodeficiency virus; PAN, polyarteritis nodosa; WG, wegener's granulomatosis; TA, takayasu arteritis; CK, creatinine kinase; ANCA, anti-neutrophil cytoplasmic antibody; pANCA, perinuclear ANCA; cANCA, classic ANCA; PR3, ptoteinase3; ESR, erythrocyte sedimentation rate; CRP, c reactive protein; NSAID, non steroidal anti-inflammatory drug; ACE, angiotensin converting enzyme; CNS, central nervous system; EULAR/PReS, european league against rheumatism/peditric rheumatology european socirty; IV IgG, intravenous immunoglobulin G

\section{Introduction}

Diagnosis of vasculitis in children is challenging because of its protean clinical manifestations with a wide spectrum ranging from isolated cutaneous vasculitis to multisystem involvement. Several medical conditions that resemble the presentation of vasculitis. There is wide array of differential diagnosis. It may be primary or secondary to various medical diseases. The secondary form must be differentiated from the primary because the treatment modalities are different. Some are benign and self limiting while others are potentially life threatening with vital organ dysfunction. So, a rational approach is mandatory for the evaluation of a child with vasculitis. This review mainly focuses on the primary vasculitis in children. There is no test to confirm the diagnosis of primary vasculitis. The diagnosis relies upon the specific criteria and exclusion of the secondarya causes of vasculitis.

Vasculitis means inflammation of the blood vessel wall. Any type of blood vessel in any organ could be affected. ${ }^{1}$ This vasculitis is characterized by fibrinoid necrosis, thrombosis and sometimes granulomatous reaction. ${ }^{2}$ Vascular damage may occur venules, capillaries, and arterioles causing local and systemic clinical manifestations, depending on the organ involvement. ${ }^{3}$ Systemic manifestations results from release of chemical mediators from inflamed blood vessels. They include fever, night sweats, malaise, weight loss, arthralgia, myalgia and laboratory features such as normocytic normochromic anaemia, leucocytosis, thrombocytosis and raised erythrocyte sedimentation rate (ESR) and $\mathrm{C}$ reactive protein (CRP). ${ }^{1}$ Treatment of some form of vasculitis secondary to infection or drugs is different from that of primary vasculitis. It is important to exclude such conditions that are likely to cause secondary vasculitis. Primary vasculitis is classified according to the size of the vessels affected.

\section{Secondary causes of vasculitis (modified from gross et al. ${ }^{4}$ )}

Inflammatory diseases of unknown aetiology: Rheumatoid vasculitis, Vasculitis associated with systemic lupus erythematosus and sjogrens syndrome, Inflammatory bowel disease, Sarcoidosis

Infectious disease: Hepatitis $\mathrm{B}$ and $\mathrm{C}$, human immunodeficiency virus, mycobacteria, syphilis

Neoplasia: hematologic malignancies such as myeloproliferative and lymphoproliferative disorders, solid tumours

Drugs: (almost any drugs) such as hydralazine, propylthiouracil, and montelukast.

Important investigations to consider during initial evaluation of patients with suspected vasculitis (depending on clinical presentation)'

\section{To exclude vasculitis "mimics" and secondary causes}
i. Blood cultures
ii. Echocardiogram
iii. Hepatitis screen (B and C)
iv. HIV test
v. Antiglomerular basement membrane antibody
vi. Antiphospholipid antibodies
vii. Antinuclear antibody

\section{To assess extent o vasculitis}

i. Urine dipstick and microscopy (all patients)

ii. Chest radiography (all patients)

iii. Nerve conduction studies/electromyography/CK

\section{To identify the specific type of vasculitis}

\author{
i. ANCA \\ ii. Cryoglobulin \\ iii. Complement levels
}

iv. Eosinophil counts/IgE levels

v. Specific findings on biopsy (necrotizing granulomatous inflammation; presence of IgA deposits, evidence of immune complex formation (or its absence) 


\section{Classification of primary vasculitis on the basis of size of affected blood vessel' \\ Large vessel vasculitis
i. Giant cell arteritis
ii. Takayasu arteritis

\section{Medium vessel vasculitis}

\section{i. Polyarteritis nodosa}

ii. Kawasaki disease

\section{Small vessel vasculitis}
i. Wegener's granulomatosis
ii. Churg-Strouss syndrome
iii. Microscopic polyangitis
iv. Henoch-Schonlein purpura
v. Cutaneous leucocytoclastic angitis
vi. Cryoglobulinaemic vasculitis

\section{Henoch-schonlein purpura (HSP)}

Henoch- Schonlein Purpura is the commonest vasculitis in childhood. HSP is found in all age groups from the age of a few months to late childhood but it is more common in young children with over $50 \%$ under 5years and over $75 \%$ under 10 years. The reported incidence of HSP varies from 10-20.4 per 100000 children.

The EULAR/PReS committee finally set criteria being published in 2010 for the diagnosis of $\operatorname{HSP}^{6}$ (Figure 1).

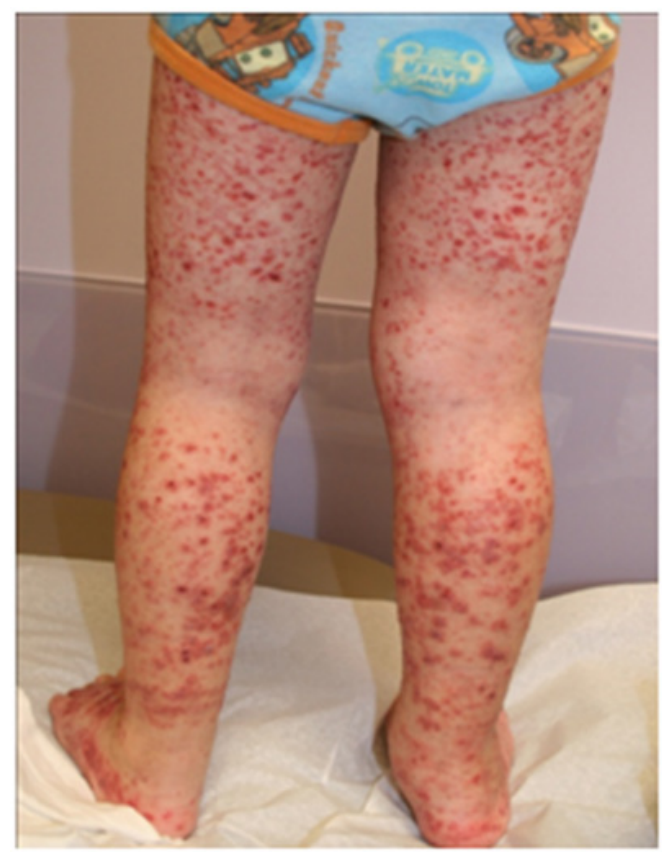

Figure I Showing feature of HSP.

Purpura (not related to thrombocytopenia), commonly palpable, with lower limb predominance (mandatory) in the presence of at least one of four features:

\#not related to thrombocytopenia a. Diffuse colicky abdominal pain, acute onset

b. Any biopsy (skin or renal) showing predominant IgA deposition

c. Arthritis or arthralgia of acute onset

d. Renal involvement: proteinuria $>0.3 \mathrm{~g} / 24 \mathrm{~h}$ or $>30 \mathrm{mmol} / \mathrm{mg}$ of urine albumin/creatinine ratio (morning sample) or hematuria or casts

The above criteria have been shown to have a sensitivity of $87.1 \%$ and a specificity of $87.7 \%{ }^{7}$

\section{Management}

Gastrointestinal symptoms may be relieved by corticosteroids $1-2 \mathrm{mg} / \mathrm{kg}$ per day when surgical conditions such as intussusceptions have been excluded. However the symptoms will resolve in most cases within a few days without the use of steroids. ${ }^{8}$ For severe gastrointestinal haemorrhage intravenous methylprednisolone has been shown to be successful. ${ }^{5}$

For management of joint problems like arthralgia/arthritis NSAID's may be used but with great caution because of potential adverse effect on gastrointestinal lining and kidneys although it lasts only a few days. Skin involvement usually does not require treatment. There is report of the use of mizoribine a purine biosynthesis inhibitor similar to mycophenolate mofetil for the management of recalcitrant purpura of HSP in an adult. ${ }^{9}$

The management of nephritis is important and careful surveillance of the urine is mandatory in all children with HSP. Since $5 \%$ of children with HSP will have serious long term sequelae. A meta-analysis reported in 2009 showed the early use of corticosteroids lessened the chance of persistent renal disease developing, whilst also diminishing the prevalence of intussusceptions and recurrence of HSP. ${ }^{10}$

There is also controversy and conflicting results regarding use of cyclophosphamide and ACE inhibitor for the improvement of outlook in established renal disease in HSP. ${ }^{11,12}$

\section{Prognosis}

HSP is usually monophasic and self-limiting. About one third have recurring disease, and those with renal disease are more likely to have recurrences which may occur up to 18 months after initial episode. ${ }^{13}$ There is a mortality of $<1 \%$ in HSP mostly due to renal involvement. ${ }^{14}$ Rarely there can be associated problems with lungs and CNS and prove to be very difficult to manage.

\section{Kawasaki disease}

KD mimics many infectious and noninfectious diseases. KD varies in its incidence worldwide and in North East Asian it is 20times more common than white children. This acute febrile illness of early childhood occurs up to $80 \%$ between 6months and 5years of age. The prevalence varies widely around world in different racial groups, which would suggest a genetic predisposition of the disease. KD occurs in 75-125 per 100000 Japanese children. Incidence in India is $14.6 / 100000$. KD is the second important cause of vasculitis in children after HSP. ${ }^{15}$

\section{Criteria for the diagnosis of Kawasaki disease. EULAR/ PreS endorsed consensus 16 (Figure 2,3 ).}

Fever persisting for at least five days (mandatory criteria) plus four of the following five features:

- Changes in peripheral extremities or perineal area 
- Polymorph ous exanthema

- Bilateral conjunctival injection

- Changes of lips and oral cavity: injection of oral and pharyngeal mucosa

- Cervical lymphadenopathy

*In the presence of coronary artery involvement (detected on echocardiography) and fever, fewer than four of the remaining five criteria are sufficient.
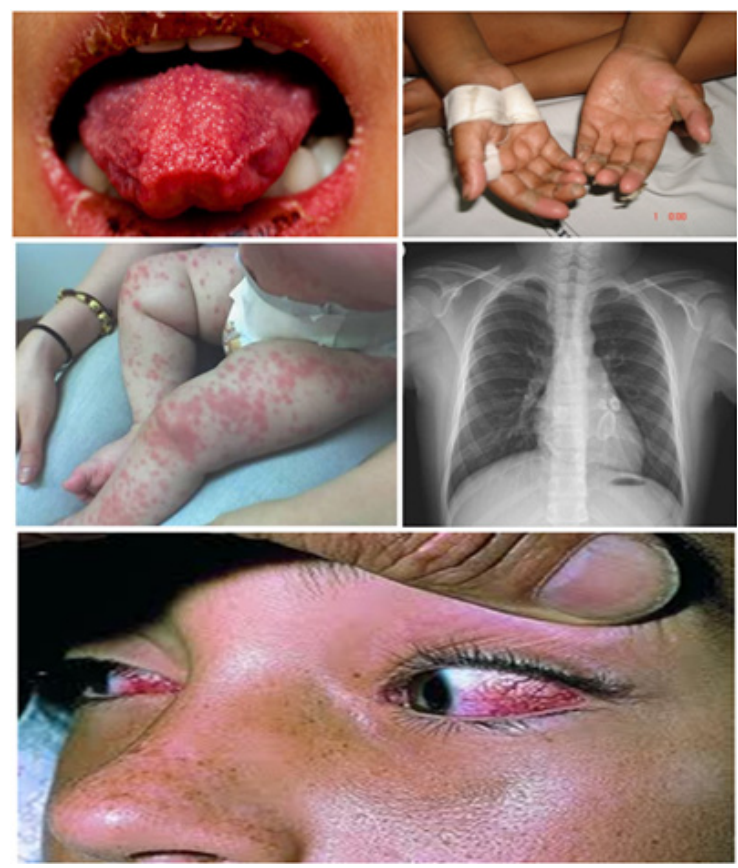

Figure 2 Showing clinical and radiological features KD.
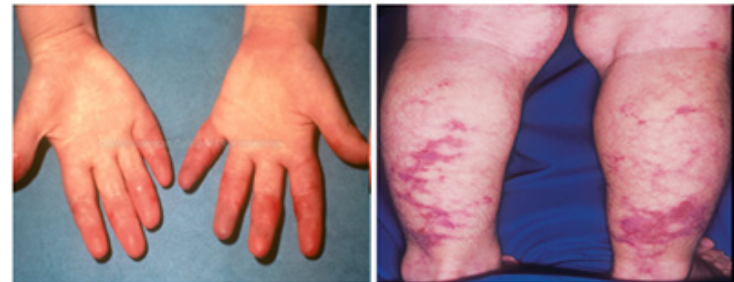

Figure 3 Showing features of PAN.

\section{Investigation}

a. CRP and ESR significantly increased and remain raised for 6-10weeks

b. Thrombocytosis $>500000$ to $1000000 / \mathrm{mm} 3$

c. Altered plasma lipids: Decreased cholesterol, decreased highdensity lipoprotein, decreased apolipoprotein

d. Mild to moderate increase serum transaminases

e. Increase bilirubin

f. Sterile pyuria

g. Hypoalbuminemia

\section{Treatment}

Intravenous immunoglobulin (IVIG) $2 \mathrm{~g} / \mathrm{kg}$, Asprin $20-25 \mathrm{mg} / \mathrm{kg}$ per dose 6hourly tried after the initial diagnosis. There is algorithm for the treatment of KD. Other drugs used are pulse methyl prednisolone, Infliximab in difficult cases. Aspirin in low doses of $3-5 \mathrm{mg} / \mathrm{kg}$ per day (anti-platelet effect) is used when fever settled for 48 hours to prevent coronary complications. ${ }^{15}$

\section{Prognosis}

KD shock syndrome has been observed when there is sustained decrease in systolic pressure of $>20 \%$ of baseline or clinical signs of poor perfusion. This syndrome is with poor outcome., and likely to be associated with the presence of coronary artery aneurysms, mitral regurgitation and myocardial dysfunction. ${ }^{17}$

\section{Childhood polyarteritis nodosa}

Polyarteritis nodosa (PAN) is a systemic vasculopathy characterized by necrotising arteritis of predominantly medium sized vessels leading to micro-aneurysms, rupture and hemorrhages manifesting with multi-system disease. The involvement of mediumsized arteries leads to pathology in skin, kidney, peripheral nerves muscle and gastrointestinal tract. ${ }^{18}$

PAN is a rare disease, and has a reported prevalence of 6.3/100000 and an incidence of 0.7/100000 in adults. In children it occurs rarely.

\section{Criteria for the diagnosis of childhood PAN6 (Figure 3)}

A systemic illness characterised by the presence of either a biopsy showing small and mid-sized artery necrotising vasculitis or angiographic abnormalities* (aneurysm or occlusion) not due to familial mediterranean fever (mandatory criteria), plus at least one of the following:

- Skin involvement (livedo reticularis, tender subcutaneous nodules, other vasculitic lesions)

- Myalgia or muscle tenderness

- Systemic hypertension, relative to childhood normative data

- Mononeuropathy or polyneuropathy

- Abnormal urine analysis and/or impaired renal function\#

- Testicular pain or tenderness

- Signs or symptoms suggesting vasculitis of any other major organ system (gastrointestinal, cardiac, pulmonary or central nervous system)

*Should include conventional angiography if magnetic resonance angiography is negative

\#Proteinuria $>0.3 \mathrm{~g} / 24$ hours, $>30$ albumin/creatinine ratio: Glomerular filtration rate of less than 50\% normal for age: haematuria or casts in urine.

\section{Investigation}

There are no specific tests for PAN. Very few children have been reported to have hepatitis B surface antigen present, as is found in adult. Elevated acute phase reactants are a constant feature. ${ }^{19}$ The presence of factor VIII-related antigen and $\beta$-thromboglobulin reflects vascular inflammation and may be used to assist in monitoring disease activity. This is regularly associated with the presence of ANCA.

\section{Treatment}

The all patients with PAN is treated with corticosteroids and also treated with cyclophosphamide. Azathioprine is used later as 
maintenance therapy. Sometimes Methotrexate and Cyclosporine is used in the treatment of PAN. IVIG is also tried in some cases. The other drugs like infliximab and rituximab is also tried in PAN. ${ }^{20}$

\section{Prognosis}

PAN has overall a good prognosis when treated early, but poor prognosis if not treated. Some has severe gastrointestinal hemorrhage and sepsis. End stage renal disease is also observed in patient with PAN. ${ }^{21}$

\section{Wegener's granulomatosis (granulomatosis with polyangitis)}

Wegener's granulomatosis (WG) is a necrotizing predominantly small-vessel vasculitis associated with granulomatous inflammation, pauci-immune necrotizing crescentic glomerulonephritis, and in most patients, the presence of antineutrophil cytoplasmic antibodies (ANCAs). ${ }^{22}$ The vasculitis has the predilection for upper airways, kidneys and lungs. It has been shown that T-cells contribute to disease mechanism. This condition occurs at all ages, with onset in childhood or adolescence in $3.3 \%$ of cases with the prevalence in this age group of the order of 0.1 per 100,000 persons. ${ }^{23}$

\section{Classification criteria for Wegener's granulomatosis}

The EULAR/PRINTO/PReS recommendation ${ }^{6}$ (Figure 4)

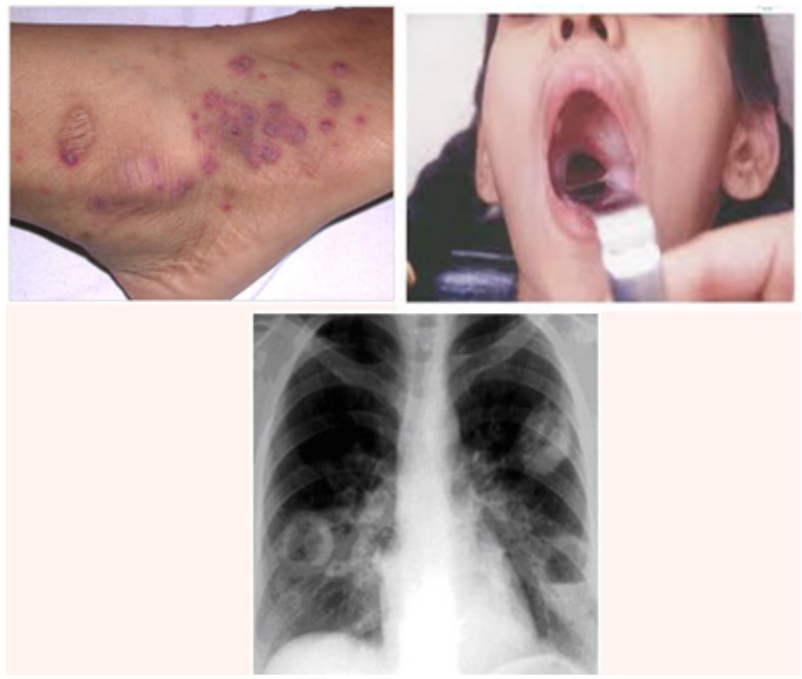

Figure 4 Showing clinical and radiological features of WG.

Three of the following six features should be present

- Abnormal urinalysis*

- Granulomatous inflammation on biopsy

- Nasal sinus inflammation

- Subglottic, tracheal, or endobronchial stenosis

- Abnormal chest X-ray or CT

- PR3 ANCA or C-ANCA staining

*Haematuria and/or significant proteinuria.

If a kidney biopsy done, it characteristically shows necrotizing pauci-immune glomerulonephritis

ANCA: Antineutrophil cytoplasmic antibodies

\section{Investigation}

There may be elevation of white cell count. There is mostly significant elevation of inflammatory markers, presence of ANCA usually c-ANCA but p-ANCA also. There is RF present in 50\%. The urine may have hematuria, proteinuria and casts in $50 \%$. There may be hypercoagulability, positive anti-phospholipid antibody and factor $\mathrm{V}$ Leiden mutations. About two thirds may have abnormal chest x-rays showing nodules, cavitations and infiltrates. ${ }^{24}$

\section{Treatment}

Traditional treatments have been gluccorticoids with the addition of oral cyclophosphamide $2 \mathrm{mg} / \mathrm{kg}$ /day for one year post remission, which usually occurs after 6 months. Intravenous cyclophosphamide is also used increasingly. Corticosteroids and methotrexate have been used in those who are intolerant of cyclophosphamide or who has less life threatening disease. Other drugs used for this disease are Mycophenolate mofetil, leflunomide and infliximab.

\section{Prognosis}

Untreated, WG has a high mortality and morbidity. With current treatment more than $91 \%$ will improve clinically, and $75 \%$ will enter clinical remission. The disease tends to flare in $60-80 \%$. Many cases survive long term but may have residual damage..$^{25,26}$

\section{Takayasu arteritis}

Takayasu arteritis (TA) is a chronic inflammatory disease characterized by granulomatous panarteritis of the aorta and main branches. It is due to cell-mediated inflammation. It has a peak incidence in third and fourth decade of life, but children are also vulnerable. It is commonly observed in the Asian children particularly Japanese. Females are more likely to be affected than males.

\section{Classification criteria for Takayasu arteritis ${ }^{6}$ (Figure 5)}

Angiographic abnormalities (conventional CT, or MR) of the aorta or its main branches (mandatory criterion), plus at least one of the following 5 features:

- Decreased peripheral artery pulse (s) and/or claudication of extremities

- Blood pressure limb discrepancy $>10 \mathrm{~mm} \mathrm{Hg}$

- Bruits over aorta and/or its major branches

- Hypertension (related to childhood normative data)

- Acute phase reactants: ESR $>20 \mathrm{~mm}$ or CRP elevated
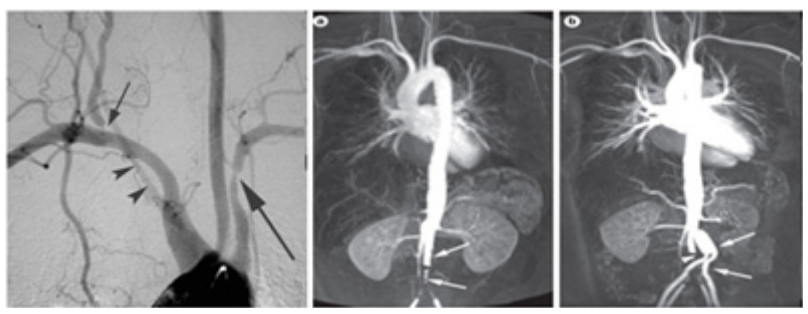

Figure 5 Showing angiographic features of TA.

\section{Treatment}

Recommended treatments for TA include steroids, cyclophosphamide, methotrexate, azathioprine, mycophenolate mofitil and anti-tumor necrosis factor- $\alpha$ blocking agents and 
antihypertensive medication as necessary. There are no controlled trials to prove guidelines for treatment strategies. Most patients respond to corticosteroid and $60 \%$ reported as attaining remission. ${ }^{27,28}$

\section{Prognosis}

Takayasu arteritis is a rare condition of children. Untreated it is uniformly fatal, and hence must be considered in sick children with PUO or unexplained inflammatory markers.

\section{Conclusion}

Child with suspected vasculitis must be medically evaluated. Secondary vasculitis has to be differentiated from the primary disease because most primary form of vasculitis consists of potentially toxic immunosuppressive therapy. Early detection and aggressive therapy is mandatory for better survival of children suffering from primary vasculitis.

\section{Acknowledgments}

None.

\section{Conflicts of interest}

Author declares there are no conflicts of interest.

\section{Funding}

None.

\section{References}

1. Suresh E. Diagnostic approach to patients with suspected vasculitis Postgrad Med J . 2006;82(970):483-488

2. Iglesias-Gamarra A. Vasculitis refractarias: aspectos generales. Rev Col Reumatol . 1999;6:144-160.

3. Iglesias-Gamarra A, Restrepo JF, Matteson EL. Small-vessel Vasculitis. Current Rheum Rep. 2007;9(4):304-311.

4. Gross Wl, Trabandi A, Reinhold-keller E. Diagnosis and evaluation of vasculitis. Rheumatology (Oxford). 2000;39(3):245-252.

5. Tizard EJ, Hamilton-Ayres MJJ. Henoch-Schonlein purpura. Arch Dis Chil Educ Prac Ed. 2008;93(1):1-8.

6. Ozen S, Pistorio A, Lusan S. EULAR/PRINTO/PreS criteria for HenochSchonlein purpura, childhood polyarteritis nodosa, childhood wegener granulomatosis and childhood Takayasu arteritis: Ankara 2008. Part II: Final classification criteria. Ann Rheum Dis . 2011;69(5):798-806.

7. Penny K, Fleming M, Kazmierczak D, et al. An epidemiological study of Henoch-Schonlein purpura. Pediatri Nurs. 2010;22(10):30-35.

8. Rosenblum N, Winter H. Steroid effects on the course of abdominal pain in children with Henoch-Schonlein purpura. Pediatrics. 1987;79(6):1018-1021.

9. Kawakami T, Shirai S, Kimura K, et al, Successful use of mizoribine to treat recurrent corticosteroid-resistant palpable purpura in a patient with Henoch-Schonlein purpura nephritis. Arch Dermatol. 2010;146(2): $212-213$.

10. Weiss PF, Feinstein JA, Luan X, et al. Effects of corticosteroid on Henoch-Schönlein purpura: a systematic review. Pediatrics. 2007;120(5):1079-1087.
11. Tarshish P, Bernstein J, Edelmann CM. Henoch-Schonlein nephritis: course of disease and efficacy of cyclophospamide. Pediar Nephrol. 2004;19(1):51-56.

12. O'Neil KM. Progress in pediatric vascultis. Curr Opin Rheumatol. 2009;21(5):538-546.

13. Calviño MC, Llorca J, García-Porrúa C, et al. Henoch-Schonlein purpura in children from Northwestern Spain. Medicine (Baltimore). 2001;80(5):279-290.

14. Tizard EJ, Hamilton-Ayres MJ. Henoch-Schonlein purpura. Arch Dis Child Educ Pract Ed. 2007;93(1):1-8.

15. Rashid AKMM, Kamal SM, Ashrafuzzaman M, et al. Kawasaki Disease and Its Treatment-An Update. Curr Rheumatol Rev. 2014;10(2): 109-116.

16. Ozen S, Ruperto N, Dillon MJ, et al. EULAR/PReS endorsed consensus criteria for the classification of childhood vasculitides. Ann Rheum Dis . 2006;65(7):936-941.

17. Kanegaye JT, Wilder MS, Molkara D, et al. Recognition of Kawasaki Shock Syndrome. Pediatrics. 2009;123(5):e783-e789.

18. Mogale KD, Shrivastava A. Childhood polyarteritis nodosa: a clinical diagnosis. Indian Pediatrics . 2006;43(10):911-913.

19. Ozen S, Anton J, Arisoy N, et al. uvenile polyarteriis: results of multicnter survey of 110 children. J Pediatr . 2004;145(4):517-522.

20. Sonomoto K, Miyamura T, Watanabe H, et al. A case of polyarteritis nodosa successfully treated by rituximab. Nihon Rinsho Memeki Gakkai Kaishi . 2008;31(2):119-123.

21. Kendirli T, Yüksel S, Oral M, et al. Fatal polyarteritis nodosa with gastrointestinal involvement in a child. Pediatr Emerg Care. 2006; 22(12):810-812.

22. Akikusa JD, Schneider R, Harvey EA, , et al. Clinical Features and Outcome of Pediatric Wegener's granulomatosis. Arthritis \& Rheumatism 2007;57(5):837-844.

23. Cotch MF, Hoffman GS, Yerg DE, et al. The epidemiology of Wegener's granulomatosis. Estimates of the five- year period prevalence, annual mortality, and geographic disease distribution from population-based data sources. Arthritis Rheum. 1996;39(1):87-92.

24. Fauci AS, Haynes BF, Katz P, et al. Wegener's granulomatosis prospective clinical and theraputic experience with 85 patients for 21 years. Ann Intern Med . 1983;98(1):76-85.

25. Fauci AS, Haynes BF, Katz P, et al. Wegener's granulomatosis. Am J Med. 1954;17:168-1679.

26. Seo P, Min YI, Holbrook JT, et al. Damage caused by Wegener's granulomatosis and its treatment: Prospective data from the Wegner's granulomatosis etaercept trial (WGET). Arthritis Rheum. 2005;52(7): $2168-2178$.

27. Mason JC. Takayasu arteritis-advances in diagnosis and management. Nat Rev Rhumatol . 2010;6(7):406-415.

28. Ishikawa K, Metani S. Long-term outcome for 120 Japanese patients with Takayasu's disease. Clinical and statistical analyses of related prognostic factors. Circulation . 1994;90(4):1855-1860. 